\title{
Challenges in identification of enteroinvasive Escherichia coli and Shigella spp. in Lebanon
}

\section{Acta Microbiologica et Immunologica Hungarica}

67 (2020) 2, 100-106

DOI:

$10.1556 / 030.2020 .01102$

๔ 2020 Akadémiai Kiadó, Budapest

ORIGINAL ARTICLE

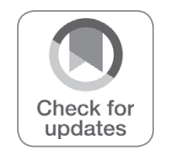

\author{
FATIMA BACHIR HALIMEH ${ }^{1,2}$, RAYANE RAFEI ${ }^{1}$, \\ SEYDINA DIENE ${ }^{2}$, MARY MIKHAEL ${ }^{1}$, HASSAN MALLAT ${ }^{1}$, \\ MARCEL ACHKAR ${ }^{3}$, FOUAD DABBOUSSI ${ }^{1}$, \\ MONZER HAMZE ${ }^{1 *}$ and JEAN-MARC ROLAIN ${ }^{2}$
}

\author{
${ }^{1}$ Laboratoire Microbiologie Santé et Environnement (LMSE), Doctoral School of Science and \\ Technology, Faculty of Public Health, Lebanese University, Tripoli, Lebanon \\ ${ }^{2}$ Aix Marseille University, IRD, APHM, MEPHI, IHU Méditerranée Infection, Faculté de Médecine \\ et de Pharmacie, 19-21 Boulevard Jean Moulin, 13385 Marseille CEDEX 05, France \\ ${ }^{3}$ Laboratory of Clinical Biology, NINI Hospital, Tripoli, Lebanon
}

Received: December 7, 2019 - Accepted: December 23, 2019

Published online: March 30, 2020

\begin{abstract}
This study aimed to evaluate the routine identification tools available in Lebanon for differentiation of Escherichia coli and Shigella spp. The identification of 43 isolates defined as Shigella spp. by Api 20E was accessed using MALDI-TOF, serological testing, duplex PCR targeting ipaH (present in Shigella spp. and enteroinvasive E. coli "EIEC") and lacY (found in E. coli including EIEC but not Shigella spp.) as well as $g y r B$ gene sequencing. Antibiotic susceptibility was investigated as well as Shiga-toxin production. All isolates were identified as E. coli by MALDI-TOF while the PCR showed a disparate group of 26 EIEC, 11 Shigella spp., 5 E. coli and 1 inactive E. coli. However, the sequencing of gyrB gene, which was recently described as a suitable marker for distinguishing E. coli and Shigella spp., identified all isolates as E. coli. Antibiotic resistance was noticeable against $ß$-lactams, rifampicin, trimethoprimsulfamethoxazole, gentamicin, and ciprofloxacin. The most common variants of beta-lactamase genes were $b l a_{\mathrm{TEM}-1}, b l a_{\mathrm{CTX}-\mathrm{M}-15}$, and $b l a_{\mathrm{CTX}-\mathrm{M}-3}$. A great discordance between the used methods in identification was revealed herein. An accurate identification technique able to distinguish E. coli from Shigella spp. in routine laboratories is a pressing need in order to select the appropriate treatment and assess the epidemiology of these bacteria.
\end{abstract}

\section{KEYWORDS}

Shigella spp., Escherichia coli, ElEC, identification, Lebanon, phenotypic techniques, molecular techniques

\section{INTRODUCTION}

Diarrheal diseases constitute a major public health issue worldwide. Children under the age of five years represent the most clinical cases, moreover gastroenteritis is ranked as the second leading cause of mortality among them, accounting for approximately 526,000 death toll in 2015 [1, 2]. Microbial and host characteristics such as the inoculum size, acidity resistance and host immunity are among factors that enhance the transmission of enteric diseases and consequently their widespread occurrence $[3,4]$.

Diarrheal diseases are caused by a variety of etiological agents (viral, parasitic, and bacterial). Among bacterial etiological agents, Escherichia coli and Shigella spp. are two of the most common cause of bacterial diarrheal diseases [5]. The genetic closeness between these organisms led many researchers to consider them as E. coli clones, albeit the distinctive morphological, biochemical, and serological features [6]. Meanwhile, Shigella spp. and EIEC (enteroinvasive E. coli) share similar genetic (analogous virulence plasmid) and biochemical features [7]. Unlike E. coli, Shigella isolates are less active biochemically and react with limited
*Corresponding author.

E-mail: mhamze@monzerhamze.com 
Table 1. The gyrB primers used for the standard PCR and sequencing

Primers

EC-SH-gyrB-F1

EC-SH-gyrB-R1

EC-SH-gyrB-F2

EC-SH-gyrB-R2

EC-SH-gyrB-F3

EC-SH-gyrB-R3
Nucleotide sequence

5'-TCACGCCGATAACTCTGTCT-3'

$5^{\prime}$-ACTCTTTCACCAGCCAGTCC- $3^{\prime}$

$5^{\prime}$-TGGCTTCCAGGAAAACATCT- $3^{\prime}$

5'-ATTTTCTGCGTCGCGTTGTA- $3^{\prime}$

$5^{\prime}$ - GTGAAATGACCCGCCGTAAA-3'

$5^{\prime}$ - TCAACAGCAGCGTACGAATG-3'
Annealing

temperature $\left({ }^{\circ} \mathrm{C}\right)$

58.54

59.6

57.02

58.86

58.84

58.93 set of antisera and shared with EIEC pathogenicity genes [8]. The high degree of relatedness was also validated by many techniques as multilocus sequence typing (MLST) and virulence genes sequencing [9]. In this context, the nomenclature of Shigella genus and its corresponding species has been kept for historical and medical reasons. Recently, the whole-genome-based, alignment-free and parameter-free CVTree approach showed that four established Shigella species form sister species to E. coli in the genus Escherichia [6]. This aforementioned contentious closeness between E. coli and Shigella spp. led to many challenges in their identification and differentiation in routine laboratories. Nowadays, many methods have been suggested to solve this dilemma as Duplex Real-Time Polymerase Chain Reaction (RT-PCR) targeting (uidA and lacY or ipaH and lacY), (MALDI-TOF MS) using an analysis software (ClinPro Tools Bruker Daltonics) [10-12] and even the Whole Genome Sequencing (WGS) followed by bioinformatics tools such as k-mers or Single Nucleotide Polymorphism [13, 14].

Similar to other developing countries, in Lebanon, the identification of Shigellosis is based on clinical manifestations and biochemical tests mainly the Api 20E gallery. Serological testing is performed only under request in specialized labs as LMSE (Laboratoire Microbiologie, Santé, et Environnement).

In our laboratory, we have a collection of 43 clinical isolates identified as Shigella spp. using Api 20E. After performing the serological and molecular analysis (PCR), our isolates are defined as a diverse group belonging to Shigella spp., EIEC, E. coli, and inactive E. coli. The aim of our study was to evaluate the used techniques in Lebanon for the identification of Shigella spp. and this by combining several molecular and phenotypic methods.

\section{MATERIALS AND METHODS}

\section{Sample collection}

A total of 43 isolates identified as Shigella spp. by API $20 \mathrm{E}$ gallery (BioMérieux, Marcy l'étoile, France) were recovered from clinical stool samples from North Lebanon between July, 2010 and September, 2016 (NINI Hospital). These isolates were conserved at the Laboratoire Microbiologie Santé et Environnement (LMSE).

\section{Phenotypic, serological and molecular identification}

Besides API 20E performed in the LMSE laboratory, identification was also ensured by MALDI-TOF MS (Bruker, Massachusetts, United States). Moreover, agglutination tests were made using Bio-Rad Antiserum antibodies (BIO-RAD, Marnes-la-Coquette, France). Molecular identification was performed by detecting two genes; ipaH (invasion plasmid antigen $\mathrm{H}$ coding gene) present in Shigella spp. and EIEC pathovar; and lacY (a lactose permease coding gene) present in the fermentative bacteria as $E$. coli [12]. The reference strains E. coli CSURP1570 [15] and Shigella flexneri DSMZ (DSM 4782-0317-001) were used as controls. Taking into account the similar species resolution of $g y r B$ sequencing and the WGS [16], we designed two couples of primers for a conventional PCR and sequencing of gyrB for the identification of our isolates (Table 1). The DNA was extracted using the BioRobot EZ1 Advanced XL instrument (Qiagen, Hilden, Germany) according to the manufacturer's instructions. A standard conventional PCR was carried out using EC-SH-gyrB-F1 and EC-SH-gyrB-R1 as external primers and the master mix QuantiTect Probe PCR Kit (QIAGEN, Hilden, Germany). The amplified fragment of $2 \mathrm{~kb}$ total size is a conserved region between E. coli and Shigella isolates. Positive PCR products were purified by NucleoFast 96 PCR plate (Machery-Nagel EURL, France) and sequenced by BigDye terminator (California, United States) and EC-SHgyrB-F2, EC-SH-gyrB-R2, EC-SH-gyrB-F3, and EC-SH-gyrB$\mathrm{R} 3$ as internal specific primers (Table 1).

\section{Shiga toxin molecular and enzyme immunoassay detection}

Enzyme immunoassay SHIGA TOXIN QUIK CHEK (Alere $^{\mathrm{TM}}$, TECHLAB ${ }^{\circledR}$, Blacksburg, United States) was used to detect the STX using specific STX1 and STX2 antibodies according to the manufacturer's instructions. Real TimePCR detecting stx 1 and stx 2 genes were carried out [17].

\section{Antimicrobial susceptibility testing}

The antimicrobial susceptibility testing was determined using the disk diffusion method on Mueller-Hinton agar, and the results were interpreted according to the EUCAST (European Committee on Antimicrobial Susceptibility Testing) [18]. The antibiotics tested were: Amoxicillin (AMX), Amoxicillin-clavulanic acid (AMC), Ticarcillin- 
A

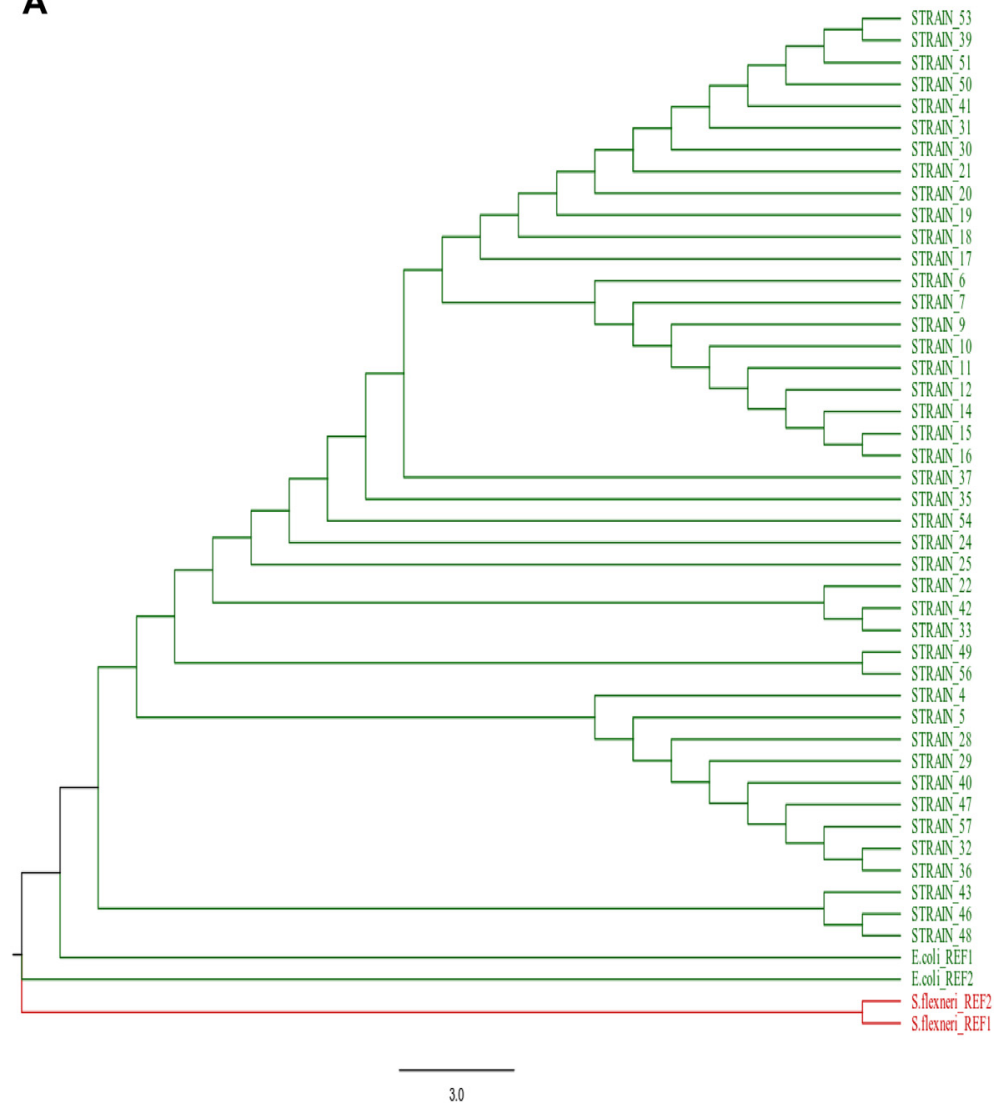

B

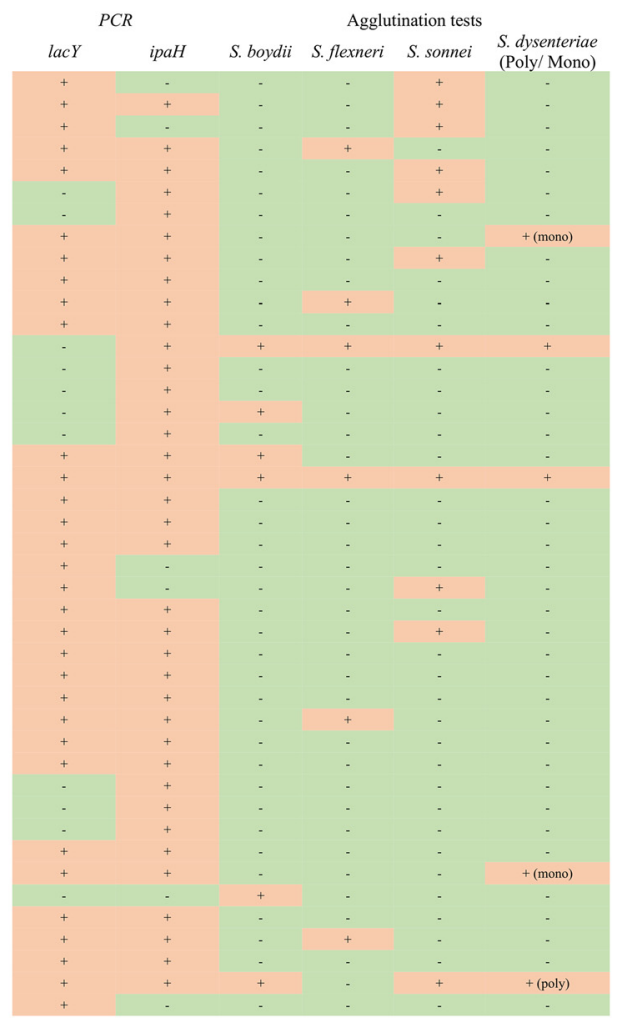

Figure 1. A: The Maximum Likehood phylogenetic tree of GyrB peptide sequences for the 43 isolates using MEGA 7 software with standard settings and 100 Bootstraps. The control strains (S. flexneri REF1) Shigella flexneri DSMZ (DSM 4782-0317-001) and (E. coli REF1) E. coli CSURP1570 he 2 NCBI reference strains (S. flexneri REF2) Shigella flexneri 2a_str_301_NC_0042272 and (E. coli REF2) Escherichia coli_str_k12_subtr_MG1655_NC_0009133 were also analyzed. FigTree V1.4 was used to modify color of branches. B: The table represents the PCR detecting lacY and ipaH genes, faced to agglutination using antisera to determine the species of isolates

clavulanic acid (TCC), Cefotaxime (CTX), Cefoxitin (FOX), Aztreonam (ATM), Ertapenem (ETP), Imipenem (IMP), Trimethoprim-Sulfamethoxazole (SXT), Rifampicin (RIF), Ciprofloxacin (CIP), Gentamicin (GN), Fosfomycin (FF), Amikacin (AK), and Colistin (CT). The phenotypic confirmation of ESBL (Extended-spectrum $\beta$-lactamase) production was performed by the double-disk synergy test (DDST).

\section{Detection of $\beta$-lactam resistance genes}

The presence of $\beta$-lactamase genes $\left(b l a_{\mathrm{TEM}}, b l a_{S \mathrm{HV}}\right.$, and bla $\left.a_{\text {CTX-M }}\right)$ was detected by PCR using specific primers for: $b l a_{\mathrm{CTX}-\mathrm{M}-1}$ group [19], bla $a_{\mathrm{CTX}-\mathrm{M}-9}$ group [20], bla $a_{\mathrm{TEM}}$ [21], $b l a_{\mathrm{SHV}}[22]$ followed by sequencing.

\section{RESULTS}

\section{Bacterial identification}

All isolates were identified as Shigella spp. using the Gallery API 20E. The agglutination tests confirmed the Shigella identity for 17 isolates where 8, 3, 4, and 2 isolates reacted with Shigella sonnei, Shigella boydii, S. flexneri, and Shigella dysenteriae antisera respectively. Additionally, 2 isolates agglutinated with all species antisera, 1 isolate cross-reacted with S. boydii, S. sonnei, and S. dysenteriae antisera, and 23 isolates didn't react with any antisera. However, all were identified as E. coli by MALDI-TOF with a high score (2.212.46). On the other hand, the duplex PCR detecting lac $Y$ and $i p a H$ revealed a heterogeneous collection of different species composed as follows: 26 isolates considered as EIEC (lacY positive, ipaH positive), 11 isolates as Shigella spp. (lacY negative, ipaH positive), 5 isolates as $E$. coli (lacY positive, $i p a H$ negative) and one isolate as inactive E. coli (lacY negative, $i p a H$ negative). This guides us to sequence the $g y r B$ gene using our own designed primers. The gyrB gene sequencing demonstrated that all isolates were $E$. coli. The $S$. flexneri and E. coli control isolates were clearly distinguished. The gyrB gene similarity ranged from $96 \%$ to $98 \%$ between $S$. flexneri control isolates and the 43 isolates, while it was $99 \%$ between $E$. coli control isolate and our isolates. Moreover, the phylogenetic tree (Fig. 1A) shows a big cluster encompassing the 43 isolates with the E. coli control isolate and a NCBI reference E. coli (Escherichia_coli_str._K12_substr._MG1655_NC_000913.3), while the S. flexneri positive control isolate is clustered with another Shigella isolate 
Table 2. Antimicrobial resistance profiles and the $\beta$-lactamase encoding genes of studied isolates

\begin{tabular}{|c|c|c|c|c|}
\hline \multirow[b]{2}{*}{ Number of isolates } & \multirow[b]{2}{*}{ Resistance Profile } & \multicolumn{3}{|c|}{$\boldsymbol{\beta}$-lactamase encoding genes } \\
\hline & & $b l a_{\mathrm{CTX}-\mathrm{M}-15}$ & $b l a_{\mathrm{CTX}-\mathrm{M}-3}$ & $b l a_{\mathrm{TEM}-1}$ \\
\hline 2 isolates & AZT, AMC, CTX, TIM, RIF, GN & + & - & + \\
\hline 2 isolates & $\begin{array}{l}\text { AMX, AZT, AMC, CTX, TIM, SXT, RIF, } \\
\text { GN }\end{array}$ & + & - & + \\
\hline 2 isolates & AMX, AZT, AMC, CTX, TIM, SXT, RIF & - & + & - \\
\hline 1 isolate & AMC, CTX, TIM, SXT, CIP, RIF & - & + & - \\
\hline 1 isolate & AMC, SXT, RIF & - & - & + \\
\hline 1 isolate & AZT, AMC, CTX, TIM, SXT, RIF, GN & + & - & + \\
\hline 1 isolate & AMX, AMC, TIM, RIF & - & - & + \\
\hline 1 isolate & AMX, AMC, FOX, TIM, SXT, CIP, RIF & - & - & + \\
\hline 1 isolate & $\begin{array}{c}\text { AMX, AZT, AMC, CTX, FOX, TIM, } \\
\text { SXT, RIF, GN }\end{array}$ & + & - & + \\
\hline 1 isolate & $\begin{array}{c}\text { AMX, AZT, AMC, CTX, FOX, SXT, CIP, } \\
\text { RIF }\end{array}$ & + & - & - \\
\hline
\end{tabular}

\section{from NCBI} NC_004337.2).

\section{Shiga toxin detection}

The immunochromatoghraphy test revealed that none of the isolates was harbored neither STX1 nor STX2, and this result is confirmed by the real time-PCR showing a negative result for both genes (stx1 and stx2).

\section{Antibiotic susceptibility results}

Most of the isolates showed reduced susceptibility to $\beta$-lactam antibiotics where $100 \%, 37.2 \%, 34.5 \%, 30.2 \%, 20.9 \%$, and $18.6 \%$ were resistant to amoxicillin-clavulanic acid, ticarcillin-clavulanic acid, amoxicillin, cefotaxime, cefoxitin, and aztreonam, respectively. In addition, rifampicin resistance was detected in $86 \%$ of isolates, trimethoprimsulfamethoxazole resistance in $79 \%$ of isolates, gentamicin resistance in $14 \%$ of isolates, and ciprofloxacin in $7 \%$ of isolates. None of these isolates was resistant to carbapenem, amikacin, fosfomycin or colistin.

\section{$\beta$-lactam resistance mechanisms}

The double-disk synergy test (DDST) detected an ESBL production in $44.1 \%(19 / 43)$ of isolates showing a synergy translated by the shape "Bouchon de Champagne." Of 19 isolates phenotypically tested positive for ESBL, 16 were resistant to more than 3 antibiotics and 13 (68.4\%) were ESBL positive by PCR. We detected the presence of the $b l a_{\mathrm{CTX}-\mathrm{M}-1}$ group in 10 isolates $(76.9 \%)$, and the $b l a_{\mathrm{TEM}}$ group in 8 isolates $(61.5 \%)$. None of the isolates had $b l a_{\mathrm{SHV}}$ or bla $a_{\text {CTX-M-9 }}$ groups. Approximately, $46 \%$ of isolates carried 2 bla genes, while $54 \%$ of them harbored a single bla gene. The sequencing showed that $70 \%$ of the $b l a_{\mathrm{CTX}-\mathrm{M}-1}$ group positive isolates were $b l a_{\mathrm{CTX}-\mathrm{M}-15}$ and $30 \%$ of them were $b l a_{\mathrm{CTX}-\mathrm{M}-3}$, while all the $b l a_{\mathrm{TEM}}$ group positive isolates were bla $a_{\mathrm{TEM}-1}$ (Table 2).

\section{DISCUSSION}

The close genetic relationship between Shigella spp. and E. coli is a scientific dilemma stumbling their accurate identification in the routine microbiological laboratories. Traditional biochemical and serological tests are the principal techniques used to identify these species in developing countries as Lebanon. However, many isolates as "inactive $E$. coli variants" cannot be identified using traditional or even molecular techniques such as conventional MALDI-TOF MS and 16S rRNA gene sequencing. Furthermore, Shigella isolates share their pathogenic genes with EIEC pathovar, thus complicating their clinical and laboratory diagnosis [23]. In this study, we aimed to evaluate the identification of 43 isolates identified as Shigella spp. using Api 20E and this by assessing a combination of phenotypic and molecular techniques. It's noteworthy to mention that 42 out of 43 isolates had LDC (lysine Desoxycarboxylase) negative test with Api 20E, a character considered negative in the Shigella genus. First of all, MALDI-TOF MS identified the isolates as E. coli with a high score (2.21-2.46). Indeed, the two phenotypic methods, namely Api 20E and MALDI-TOF MS, used different targets in order to unveil the identity of a particular bacterium. Gallery Api 20E's identification is based on the detection of the enzymatic activity and the fermentation of carbohydrates, while MALDI-TOF MS identifies bacteria through analysis of their proteins (mainly ribosomal proteins) in the mass range between 2,000 and 20,000 Daltons. Regarding their accuracy in identification, Api $20 \mathrm{E}$ has been qualified and preferred in many laboratories for the differentiation of Enterobacteriaceae family with a rate of correct identification reaching up to $97 \%$ [24, 25]. However, MALDI-TOF has a low-resolution power to distinguish between some taxonomic groups like E. coli and Shigella spp. [11, 26, 27]. Otherwise, a novel approaches approved by Bruker Daltonics (ClinPro Tools software), not used herein, can increase the taxonomic group resolution unachievable by methods like $16 S$ rDNA sequencing and routine MALDI-TOF MS [23, 28]. 
Due to this observed inconsistency, we have performed serological testing with traditional Shigella spp. antisera for these isolates. Serology, based on the detection of $\mathrm{O}$ antigen present in the outer membrane of Gram-negative bacteria linked to Lipopolysaccharide (LPS) backbone, has been considered till now as the reference technique for the identification of Shigella at species and serotype level. However, there were many examples of Shigella serotypes being misidentified through literature [29]. For example, $S$. flexneri serotype 6 was misidentified through history and was related phylogenetically to $S$. boydii. In addition, many $\mathrm{O}$ antigens of known Shigella serotypes are shared with some E. coli pathovars [13]. Indeed, S. boydii and S. dysenteriae share the same $\mathrm{O}$ antigen structure as that of EIEC leading thus to false positive results [13]. A recent paper unveiled the genetic causes behind the observed discordance between the traditional approach (serological testing combined with biochemical tests) and the k-mer identification derived from WGS. S. flexneri was misidentified by the traditional approach as $S$. boydii due to a dysfunctional $W Z X_{1-5}$ gene [13]. Interestingly, a great percentage of our isolates were not typeable accounting for $53 \%$ of the total, a percentage higher those reported elsewhere in the world [30, 31].

Many of molecular techniques have been proposed to discriminate Shigella spp. from E. coli. The duplex PCR amplified the lacY gene (lactose permease gene) and either uidA (beta-glucuronidase encoding gene) or ipaH (invasion plasmid antigen $\mathrm{H}$ encoding gene) [10, 12]. The first schema targets the uidA found in both species and lacY particularly found in lactose-fermenting species like E. coli. Otherwise, the second schema can differentiate between E. coli, EIEC, and Shigella spp. The lacY gene is common in both E. coli and EIEC, while the ipaH gene is found in EIEC and Shigella spp. Also, Shigella spp. lacks the lacY gene [12]. In the present study, the performed duplex PCR revealed a diverse population among our isolates composed of E. coli, EIEC, inactive E. coli, and Shigella spp. Otherwise, this method can be also criticized. First, although some Shigella spp. lack the lacY gene, S. dysenteriae has lacA and lacY genes and S. sonnei has the three genes but they are unable to ferment lactose due to the lack of the permease activity [32]. Second, ipaH is considered as a virulence factor gene exclusively located on the virulence plasmid harbored by Shigella isolates. But the existence of several putative ipaH cognate genes in the chromosome is already mentioned $[33,34]$. In this line, the presence of $i p a H$ on the plasmid could lead to false positive or negative PCR results, due to the horizontal transfer of plasmid among the Enterobacteriaceae genera [28].

Comparing molecular results with the serological test, a huge discordance was noted. For example, among 26 isolates defined as EIEC by PCR, 12 reacted with Shigella spp. antisera. Additionally, of 5 isolates considered as E. coli by PCR, 3 reacted with Shigella spp. antisera. On the other hand, within 11 isolates identified as Shigella spp. by PCR, 7 didn't have any reaction with antisera (Fig. 1B). This discordance can be explained by the presence of common $\mathrm{O}$ antigen between Shigella, EIEC, and E. coli species [13]. Notoriously, a novel Shigella pathotype ST270; even considered as Shigella by k- mers derived by WGS, was identified as EIEC by traditional biochemical and serological test [35].

In the present study, the gyrB was able to identify correctly the E. coli and Shigella control strains, but it identified all the studied isolates as $E$. coli, even with the serological and biochemical evidence. Recently, gyrB gene, which encodes the subunit $\beta$ protein of DNA gyrase (Topoisomerase type II protein), was considered as a suitable phylogenetic marker commonly used in the identification and classification of the evolutionary relationships of closely related species [36, 37]. Furthermore, a recent study demonstrated the ability of gyrB sequence in distinguishing the different Shigella isolates with percentages of divergence higher than found in $16 \mathrm{~S}$ rRNA and 23S rRNA [38]. In Shigella, only one study described the comparable ability between gyrB gene sequencing and k-mer derived from WGS to distinguish Shigella from E. coli to the species level [16]. Other studies should be conducted to assess the usefulness of this gene for the differentiation of $E$. coli and Shigella spp.

Moreover, in our study, we didn't detect any Shiga-toxinproducing isolates. Indeed, Shiga-toxin is commonly found in S. dysenteriae serotype 1 (SD1) and Shiga-Toxin E. coli. However, we had one isolate identified as $S$. dysenteriae by antisera agglutination and PCR, but it didn't produce the Shiga-toxin. This raises also substantial differences between the identification techniques.

Overall, the prevalence of ESBL-producing isolates was $30 \%$ (13/43), where $b l a_{\mathrm{TEM}-1}(69.2 \%)$ was the most common variants followed by $b l a_{\mathrm{CTX}-\mathrm{M}-15}(53.8 \%)$, and $b l a_{\mathrm{CTX}-\mathrm{M}-3}$ (23\%). In Lebanon, E. coli constitutes about $54.7 \%$ of Gramnegative bacteria isolated from hospitalized patients, of which $32.1 \%$ harbored ESBL resistance genes [39]. In congruence with the worldwide situation, the rate of ESBLproducing E. coli isolates has shown an upward trend in Lebanon from $2 \%$ in 2003 to up to $33.6 \%$ in 2013, with ongoing increasing values [39, 40]. Compared to ESBLproducing E. coli, ESBL-producing Shigella isolates are less common worldwide, nevertheless, the rates vary according to countries and fluctuate between $1.5 \%$ and $68 \%$ [41-43].

\section{CONCLUSION}

In conclusion, although our study doesn't succeed to unravel the identity of isolates, we addressed here a real problem hindering the routine identification of Shigella spp. and E. coli in clinical microbiology laboratories, especially in low-income countries. Moreover, such misidentification can affect the accurate assessment of the appropriate treatment and the epidemiology of these bacteria. Indeed, if Shigella was misidentified as an E. coli from an extra-intestinal site, the treatment would be appropriate for an E. coli isolate. However, the susceptibility testing differs between these two organisms according to the CLSI (Clinical and Laboratory Standards Institute) guide where the first and second generation cephalosporins, cephamycin, and aminoglycosides 
are not tested for Shigella isolates, as false in vitro susceptibility may occur [44]. Each used method herein seems to answer differently the tackling question, and an accurate method as WGS is highly needed to conclude about the identity of isolates and select the most appropriate method for species differentiation in low-income countries labs. One of the most helpful and rapid techniques that can be used to distinguish E. coli from Shigella spp. is Filmarray ${ }^{\mathrm{TM}}$ GI panel (BioMérieux, Marcy l'étoile, France) detecting 22 pathogens in less than $1 \mathrm{~h}$ with high sensitivity and specificity comparable to traditional laboratory methods [45].

Funding: This study was supported by the Lebanese University and the Aix-Marseille University.

\section{ACKNOWLEDGMENT}

The authors thank Taha Abdo, Mariam Yehya, Iman Darwich, and Nazih Lazkani from the Lebanese university. And Iman Dandachi and Linda Hadjadj from Aix-Marseille University.

\section{REFERENCES}

[1] WHO. By category | Number of deaths by region - diarrhoeal diseases, http://apps.who.int/gho/data/view.main.CM1002015 REG6-CH3?lang=en.

[2] Schroeder GN, Hilbi H. Molecular pathogenesis of Shigella spp.: controlling host cell signaling, invasion, and death by type III secretion. Clin Microbiol Rev 2008; 21: 134-56.

[3] Lima IFN, Havt A, Lima AAM. Update on molecular epidemiology of Shigella infection. Curr Opin Gastroenterol 2015; 31: $30-7$.

[4] Keusch GT, Walker CF, Das JK, Horton S, Habte D. Diarrheal diseases. In: Black RE, Laxminarayan R, Temmerman $M$, Walker N, editors. Reproductive, maternal, newborn, and child health: disease control priorities. 3rd ed., vol. 2. Washington (DC): The International Bank for Reconstruction and Development | The World Bank; 2016.

[5] Kotloff KL, Platts-Mills JA, Nasrin D, Roose A, Blackwelder WC, Levine MM. Global burden of diarrheal diseases among children in developing countries: incidence, etiology, and insights from new molecular diagnostic techniques. Vaccine 2017; 35: 6783-9.

[6] Zuo G, Xu Z, Hao B. Shigella strains are not clones of Escherichia coli but sister species in the genus Escherichia. Dev Reprod Biol 2013; 11: 61-5.

[7] The HC, Thanh DP, Holt KE, Thomson NR, Baker S. The genomic signatures of Shigella evolution, adaptation and geographical spread. Nat Rev Microbiol 2016; 14: 235-50.

[8] van den Beld MJC, Reubsaet FAG. Differentiation between Shigella, enteroinvasive Escherichia coli (EIEC) and noninvasive Escherichia coli. Eur J Clin Microbiol Infect Dis 2012; 31: 899-904.

[9] Yang C, Li P, Zhang X, Ma Q, Cui X, Li H, et al. Molecular characterization and analysis of high-level multidrug- resistance of Shigella flexneri serotype 4s strains from China. Sci Rep 2016; 6: 29124.

[10] Pavlovic M, Luze A, Konrad R, Berger A, Sing A, Busch U, et al. Development of a duplex real-time PCR for differentiation between E. coli and Shigella spp. J Appl Microbiol 2011; 110: 1245-51.

[11] Rahi P, Prakash O, Shouche YS. Matrix-assisted laser desorption/ionization time-of-flight mass-spectrometry (MALDI-TOF MS) based microbial identifications: challenges and scopes for microbial ecologists. Front Microbiol 2016; 7: 1359.

[12] Løbersli I, Wester AL, Kristiansen Å, Brandal LT. Molecular differentiation of Shigella spp. from enteroinvasive E. coli. Eur J Microbiol Immunol 2016; 6: 197-205.

[13] Chattaway MA, Schaefer U, Tewolde R, Dallman TJ, Jenkins C. Identification of Escherichia coli and Shigella species from whole-genome sequences. J Clin Microbiol 2017; 55: 616-23.

[14] Pettengill EA, Pettengill JB, Binet R. Phylogenetic Analyses of Shigella and Enteroinvasive Escherichia coli for the identification of molecular epidemiological markers: whole-genome comparative analysis does not support distinct genera designation. Front Microbiol 2015; 6: 1573.

[15] Al Bayssari C, Olaitan AO, Dabboussi F, Hamze M, Rolain JM. Emergence of OXA-48-producing Escherichia coli clone ST38 in fowl. Antimicrob Agents Chemother 2015; 59: 745-6.

[16] Dhiviya Prabaa MS, Naveen Kumar DR, Yesurajan IF, Anandan S, Kamini W, Balaji V. Identification of nonserotypeable Shigella spp. using genome sequencing: a step forward. Future Sci OA 2017; 3: FSO229.

[17] Bélanger SD, Boissinot M, Ménard C, Picard FJ, Bergeron MG. Rapid detection of Shiga toxin-producing bacteria in feces by multiplex PCR with molecular beacons on the smart cycler. J Clin Microbiol 2002; 40: 1436-40.

[18] EUCAST. The European committee on antimicrobial susceptibility testing, http://www.eucast.org/.

[19] Roschanski N, Fischer J, Guerra B, Roesler U. Development of a multiplex real-time PCR for the rapid detection of the predominant beta-lactamase genes CTX-M, SHV, TEM and CIT-type AmpCs in Enterobacteriaceae. PloS One 2014; 9: e100956.

[20] Edelstein M, Pimkin M, Palagin I, Edelstein I, Stratchounski L. Prevalence and molecular epidemiology of CTX-M extendedspectrum beta-lactamase-producing Escherichia coli and Klebsiella pneumoniae in Russian hospitals. Antimicrob Agents Chemother 2003; 47: 3724-32.

[21] Tajbakhsh M, Garcia Migura L, Rahbar M, Svendsen CA, Mohammadzadeh M, Zali MR, et al. Antimicrobial-resistant Shigella infections from Iran: an overlooked problem?. J Antimicrob Chemother 2012; 67: 1128-33.

[22] Yagi T, Kurokawa H, Shibata N, Shibayama K, Arakawa Y. A preliminary survey of extended-spectrum beta-lactamases (ESBLs) in clinical isolates of Klebsiella pneumoniae and Escherichia coli in Japan. FEMS Microbiol Lett 2000; 184: 53-6.

[23] Khot PD, Fisher MA. Novel approach for differentiating Shigella species and Escherichia coli by matrix-assisted laser desorption ionization-time of flight mass spectrometry. J Clin Microbiol 2013; 51: 3711-6. 
[24] Hsu BM, Wu SF, Huang SW, Tseng YJ, Ji DD, Chen JS, et al. Differentiation and identification of Shigella spp. and enteroinvasive Escherichia coli in environmental waters by a molecular method and biochemical test. Water Res 2010; 44: 949-55.

[25] Ekwall E, Dimander M. Comparison of micro-ID with API $20 \mathrm{E}$ for identification of Salmonella, Shigella and Yersinia species. Eur J Clin Microbiol 1982; 1: 134-7.

[26] Li B, Liu H, Wang W. Multiplex real-time PCR assay for detection of Escherichia coli O157:H7 and screening for nonO157 Shiga toxin-producing E. coli. BMC Microbiol 2017; 17: 215.

[27] Khot PD, Couturier MR, Wilson A, Croft A, Fisher MA. Optimization of matrix-assisted laser desorption ionizationtime of flight mass spectrometry analysis for bacterial identification. J Clin Microbiol 2012; 50: 3845-52.

[28] Kim HJ, Ryu JO, Song JY, Kim HY. Multiplex polymerase chain reaction for identification of Shigellae and Four Shigella species using novel genetic markers screened by comparative genomics. Foodborne Pathog Dis 2017; 14: 400-6.

[29] Liu B, Knirel YA, Feng L, Perepelov AV, Senchenkova SN, Wang Q, et al. Structure and genetics of Shigella O antigens. FEMS Microbiol Rev 2008; 32: 627-53.

[30] Anandan S, Muthuirulandi Sethuvel DP, Gajendiren R, Verghese VP, Walia K, Veeraraghavan B. Molecular characterization of antimicrobial resistance in clinical Shigella isolates during 2014 and 2015: trends in South India. Germs 2017; 7: 115-22.

[31] Shakya G, Acharya J, Adhikari S, Rijal N. Shigellosis in Nepal: 13 years review of nationwide surveillance. J Health Popul Nutr 2016; 35: 36.

[32] Devanga Ragupathi NK, Muthuirulandi Sethuvel DP, Inbanathan FY, Veeraraghavan B. Accurate differentiation of Escherichia coli and Shigella serogroups: challenges and strategies. New Microbes New Infect 2018; 21: 58-62.

[33] Ashida H, Sasakawa C. Shigella IpaH family effectors as a versatile model for studying Pathogenic Bacteria. Front Cell Infect Microbiol 2015; 5: 100.

[34] Ashida H, Toyotome T, Nagai T, Sasakawa C. Shigella chromosomal IpaH proteins are secreted via the type III secretion system and act as effectors. Mol Microbiol 2007; 63: 680-93.

[35] Hazen TH, Leonard SR, Lampel KA, Lacher DW, Maurelli AT, Rasko DA. Investigating the relatedness of Enteroinvasive
Escherichia coli to other E. coli and Shigella isolates by using comparative genomics. Infect Immun 2016; 84: 2362-71.

[36] Kasai H, Tamura T, Harayama S. Intrageneric relationships among Micromonospora species deduced from gyrB-based phylogeny and DNA relatedness. Int J Syst Evol Microbiol 2000; 50: 127-34.

[37] Wang LT, Lee FL, Tai CJ, Kasai H. Comparison of gyrB gene sequences, 16S rRNA gene sequences and DNA-DNA hybridization in the Bacillus subtilis group. Int J Syst Evol Microbiol 2007; 57: 1846-50.

[38] Vos M, Quince C, Pijl AS, de Hollander M, Kowalchuk GA. A Comparison of $r p o B$ and 16S rRNA as markers in pyrosequencing studies of bacterial diversity. PLoS One 2012; 7: e30600.

[39] Chamoun K, Farah M, Araj G, Daoud Z, Moghnieh R, Salameh P, et al. Lebanese Society of Infectious Diseases study group (LSID study group): surveillance of antimicrobial resistance in Lebanese hospitals: retrospective nationwide compiled data. Int J Infect Dis 2016; 46: 64-70.

[40] Daoud Z, Hakime N. Prevalence and susceptibility patterns of extended-spectrum beta-lactamase-producing Escherichia coli and Klebsiella pneumoniae in a general university hospital in Beirut, Lebanon. Rev Esp Quimioter 2003; 16: 233-8.

[41] Keddy KH, Sooka A, Crowther-Gibson P, Quan V, Meiring S, Cohen C, et al. Group for Enteric, Respiratory, and Meningeal Disease Surveillance in South Africa (GERMS-SA): systemic shigellosis in South Africa. Clin Infect Dis 2012; 54: 1448-54.

[42] Madhavan A, Balakrishnan S, Vasudevapanicker J. Antibiotic susceptibility pattern of Shigella isolates in a tertiary healthcare center. J Lab Phys 2018; 10: 140-4.

[43] Liu Y, Cheng Y, Yang H, Hu L, Cheng J, Ye Y, et al. Characterization of extended-spectrum $\beta$-lactamase genes of Shigella flexneri isolates with fosfomycin resistance from patients in China. Ann Lab Med 2017; 37: 415-9.

[44] Dekker JP, Frank KM. Salmonella, Shigella, and Yersinia. Clin Lab Med 2015; 35: 225-46.

[45] Valenzuela C, Legarraga P, Peña A, Arenas A, Berkowitz L, Ramírez G, et al. Etiologic and clinical characterization of community acquired gastroenteritis in adult patients in a Chilean emergency room by the film array GI panel. PLoS One 2018; 13: e0207850. 